\title{
Electrostatics at the Molecular Level
}

Ulrich Zurcher

Cleveland State University, u.zurcher@csuohio.edu

Follow this and additional works at: https://engagedscholarship.csuohio.edu/sciphysics_facpub

Part of the Quantum Physics Commons

How does access to this work benefit you? Let us know!

\section{Repository Citation}

Zurcher, Ulrich, "Electrostatics at the Molecular Level" (2017). Physics Faculty Publications. 376.

https://engagedscholarship.csuohio.edu/sciphysics_facpub/376

This Article is brought to you for free and open access by the Physics Department at EngagedScholarship@CSU. It has been accepted for inclusion in Physics Faculty Publications by an authorized administrator of EngagedScholarship@CSU. For more information, please contact library.es@csuohio.edu. 
PAPER

\section{Electrostatics at the molecular level}

To cite this article: Ulrich Zürcher 2017 Eur. J. Phys. 38015206

View the article online for updates and enhancements.

\section{Related content}

Structural aspects of the ice-water system $\mathrm{NH}$ Fletcher

- Close encounters with DNA C Maffeo, J Yoo, J Comer et al.

Breaking Newton's third law: electromagnetic instances

Fabiana B Kneubil 


\title{
Electrostatics at the molecular level
}

\author{
Ulrich Zürcher \\ Physics Department, Cleveland State University, Cleveland, OH 44115, USA \\ E-mail: u.zurcher@csuohio.edu
}

Received 18 August 2016, revised 14 October 2016

Accepted for publication 19 October 2016

Published 21 November 2016

\begin{abstract}
In molecular systems, positive and negative charges are separated, making them ideal systems to examine electrostatic interactions. The attractive force between positive and negative charges is balanced by repulsive 'forces' that are quantum-mechanical in origin. We introduce an 'effective' potential energy that captures the repulsion; it allows us to obtain fairly accurate estimates of the bonding properties of molecular systems. We use units (e.g., kcal $\mathrm{mol}^{-1}$ for energy) that emphasize the relevance of electrostatics to macroscopic behavior.
\end{abstract}

Keywords: electrostatics, chemical bond, thermal properties of matter

\section{Introduction}

The Next Generation Science Standards [1] that emphasizes finding solutions to real-world problems. In the standard MS-PS1-1, students are expected to 'develop models to describe the atomic composition of simple molecules and extended structures. Emphasis is on developing models of molecules that vary in complexity. Examples of simple molecules could include ammonia and methanol. Examples of extended structures could include sodium chloride or diamonds. Examples of molecular-level models could include drawings, 3D ball and stick structures, or computer representations showing different molecules with different types of atoms'.

In fact, many students, especially those in the algebra-based sequence, are first introduced to electric charges and the forces between them in a general chemistry course when the bonding of atoms in molecules is discussed. Molecular systems are compelling examples for electrostatics because Coulomb forces are dominant on the scale of nanometers and below. This is not the case on the scale of centimeters and longer, where the Coulomb force is often a 'curiosity' (e.g., 'clinging' of clothes taken from a dryer), although electric forces are billionbillion-billion-billion times stronger than gravitational forces, as Feynman points out [2]. Electric force goes largely unnoticed in every day life because the positive and negative charges are perfectly balanced. The fact that electrons and protons are kept in roughly the 
same places explains the rigidity of macroscopic material: for example, the $440 \mathrm{~m}$ Willis Tower in Chicago swings only about $1 \mathrm{~m}$ in a strong storm [3].

Physical theories should be illustrated with examples of realistic systems so that students can develop a 'sense' of numbers. The author of recent article in The Physics Teacher [4] discusses why this is a challenge for electrostatics. This explains why in many textbook problems, one encounters electric fields between charged objects that exceeds the breakdown value for air $\left(3 \times 10^{6} \mathrm{~N} \mathrm{C}^{-1}\right)$, while in other cases, the electric charge is unreasonably high (e.g., higher than the $7 \mu \mathrm{C}$-charge of a $450 \mathrm{kV}$ Van de Graff). The author proposes that values of electrostatics problems should be 'those that are readily achieved in laboratory and classroom situations'. Known examples are (party-) balloons, pith balls, styrofoam peanuts, and electroscopes.

However, it is only difficult to find macroscopic examples of electrostatics if we restrict ourselves to few-particle systems, similar to mechanical systems, but 'the phenomena we commonly observe involve not two or three but of the order of $10^{27}$ particles' [5]. For example, the latent heat of fusion of water $\left(\mathrm{H}_{2} \mathrm{O}\right) L_{\mathrm{f}}=6.04 \mathrm{~kJ} \mathrm{~mol}^{-1}$ is related to molecular properties via Avogadro's number $N_{\mathrm{A}}=6.02 \times 10^{23} \mathrm{~mol}^{-1}$ so that $\epsilon=L_{\mathrm{f}} / N_{\mathrm{A}}=$ $1.0 \times 10^{-20} \mathrm{~J}$. This calculation suggests that molecular systems can be used to illustrate the principles of electrostatics; students 'make sense of numbers' by relating the results to a macroscopic behavior of matter [4].

The description of electrons in molecules requires, of course, a quantum mechanical treatment, and we refer the reader to [6] for a rather complete treatment in the case of small molecules. Two main features emerge from such a treatment: (1) the electrons is shared by the atoms in a molecule, i.e., there is a finite probability for any electron to be near any atom, so that the 'effective' charge of an atom is a fraction of an elementary charge and can be negative or positive; we say that atoms have partial charges. (2) There is a repulsive force between atoms and molecules at short distances and essentially has quantum mechanical origin: (i) When electron shells overlap, the atomic nuclei are no longer shielded and repel each other. (ii) Electrons with the same spin repel each other due to the Pauli exclusion principle when their orbitals overlap [7]. As a result, attractive Coulomb forces are balanced by repulsive forces, which explains the stability of molecules and, in particular the equilibrium lengths of bonds.

We develop a simple model of the rigidity of the $\mathrm{OH}$-bond of a water molecule $\mathrm{H}_{2} \mathrm{O}$ and the hydrogen bonding of a water dimer. We use known values for the partial charges of hydrogen and oxygen atoms in a water molecule. We treat the repulsion between atoms by introducing a phenomenological potential that decays exponentially with the distance between atoms. Phenomenological models play an important role as they provide 'a quick practical way to form simple pictures and rough estimates of properties whose more precise comprehension may require analysis of considerable complexity' [8].

The outline of the paper is as follows. We discuss the relevant scales of the energy and force at the molecular level in section 2. We examine the intramolecular force of the $\mathrm{OH}$-bond in a water molecule in section 3, and obtain the repulsive forces between oxygen and hydrogen from the vibrational properties of the $\mathrm{OH}$-bond; we calculate the force from Coulomb's law, rather than from the derivative of the electrostatic potential energy, to make a clear connection to introductory physics. We discuss the intermolecular force between molecules in a water dimer in section 4; we use a simple argument to find the net attraction between oxygen and hydrogen atoms responsible for hydrogen bonding. We then find the bonding energy of hydrogen bond and its vibrational property. We relate the intra- and intermolecular binding energies to thermal properties of water. We summarize our results in 
section 5, and discuss some implications for the teaching of electrostatics in the algebra-based introductory physics.

\section{Energy scales}

The force between two charges $q_{1}$ and $q_{2}$ separated by the distance $r$ is given by Coulomb's law $F=\left(4 \pi \epsilon_{0}\right)^{-1} q_{1} q_{2} / r^{2}$, and the corresponding potential energy is EPE $=\left(4 \pi \epsilon_{0}\right)^{-1} q_{1} q_{2} / r$, with $\left(4 \pi \epsilon_{0}\right)^{-1}=9.0 \times 10^{9} \mathrm{Nm}^{2} \mathrm{C}^{-2}$. The appropriate length scale for atoms and molecules is one Angstrom:

$$
\mathcal{L}=1.0 \times 10^{-10} \mathrm{~m}=100 \mathrm{pm} .
$$

This length scale defines the energy scale $\mathcal{U}=\left(4 \pi \epsilon_{0}\right)^{-1} e^{2} / \mathcal{L}=2.3 \times 10^{-18} \mathrm{~J}$. In the context of chemistry and biology, (reaction) energies are often expressed in units of $\mathrm{kcal} \mathrm{mol}^{-1}: 1 \mathrm{kcal} \mathrm{mol}^{-1}$ corresponds to $7.0 \times 10^{-21} \mathrm{~J}$ per atom or molecule. We write

$$
\mathcal{U}=330 \mathrm{kcal} \mathrm{mol}^{-1} \text {. }
$$

We compare this energy with other relevant energies. The latent heat characterizes the 'chemical energy' of matter. The values for water are $L_{\mathrm{f}}=1.44 \mathrm{kcal} \mathrm{mol}^{-1}=0.0044 \mathcal{U}$ (fusion) and $L_{\mathrm{v}}=9.72 \mathrm{kcal} \mathrm{mol}^{-1}=0.092 \mathcal{U}$ (vaporization), and are thus much smaller than $\mathcal{U}$.

The thermal energy is determined by the Boltzmann constant is $k_{\mathrm{B}} T=1.38 \times 10^{-23} \mathrm{~J} \mathrm{~K}^{-1}$

$$
k_{\mathrm{B}}=0.002 \mathrm{kcal} \mathrm{mol}^{-1} \mathrm{~K}^{-1} \text {. }
$$

We calculate the corresponding temperature $T_{\mathcal{U}}=\mathcal{U} / k_{\mathrm{B}}=1.6 \times 10^{5} \mathrm{~K}$, which shows that $\mathcal{U}$ is an enormous energy for atomic and molecular systems.

Similarly, we define a characteristic force from Coulomb's law $\mathcal{F}=\left(4 \pi \epsilon_{0}\right)^{-1} e^{2} / \mathcal{L}^{2}$, or

$$
\mathcal{F}=330 \mathrm{kcal} \mathrm{mol}^{-1} \AA^{-1} \text {. }
$$

A typical external electric field is $E_{0}=1000 \mathrm{~V} \mathrm{~m}^{-1}$ so that $e E_{0}=7.0 \times$ $10^{-9} \mathrm{kcal}(\mathrm{mol} \AA)^{-1}$. We write $E=E^{\prime} E_{0}$ and thus find the force on a partial charge $\delta q$ in the electric field $E^{\prime}$ :

$$
F=7.0 \times 10^{-9} \mathrm{kcal} \mathrm{mol}^{-1} \AA^{-1} E^{\prime} \delta q .
$$

We find an upper bound of the scaled electric field from the the (average) intensity of a typical (industrial) solid-state laser $S=3 \times 10^{8} \mathrm{~W} \mathrm{~m}^{-2}$

$$
E_{\max }^{\prime}=300 \text {. }
$$

The maximum external force on a charge follows $F_{\max }=2.1 \times 10^{-6} \mathrm{kcal}(\mathrm{mol} \AA)^{-1}$, which is about six orders of magnitude smaller than the characteristic force $\mathcal{F}$.

We introduce scaled variables. We write the distance $d=\mathcal{L} d^{\prime}$, and drop the prime in the following. The potential energy follows

$$
\operatorname{EPE}(r)=330 \mathrm{kcal} \mathrm{mol}^{-1} \frac{\delta q_{1} \delta q_{2}}{r}
$$

and similarly the force:

$$
F=330 \mathrm{kcal} \mathrm{mol}^{-1} \AA^{-1} \frac{\delta q_{1} \delta q_{2}}{r^{2}} .
$$




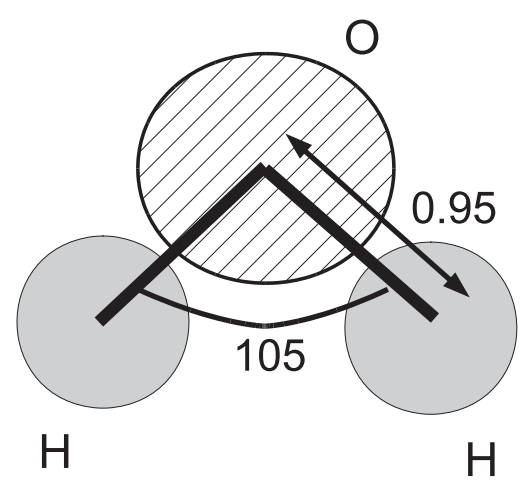

Figure 1. The model of a water molecule. The equilibrium length of the OH-bond is $l_{0}=0.95$ and the angle between the bonds is $105^{\circ}$.

\section{Intramoleculer forces}

The (covalent) atomic radii of hydrogen and oxygen are found $R_{\mathrm{H}}=0.25$ and $R_{\mathrm{O}}=0.6$; the partial charges $\operatorname{are}^{1} \delta q_{\mathrm{H}}=+0.4$ and $\delta q_{\mathrm{O}}=-0.8$ [9]. The equilibrium distance $\mathrm{OH}$ in a water molecule is $l_{0}=0.95$, and the angle between the two $\mathrm{OH}$ bonds is $105^{\circ}$, cf figure 1 . The electrostatic interaction between oxygen and hydrogen follows

$$
\operatorname{EPE}(r)=-100 \mathrm{kcal} \mathrm{mol}^{-1} \frac{1}{r} .
$$

We ignore the motion of the oxygen atom since the oxygen atom is much heavier than the hydrogen atom.

The properties of the OH-bond is determined by the electrostatic interactions and the repulsive force between charges. The net force explains the equilibrium length and the deviation from equilibrium give rise to an 'effective' elastic behavior of the OH-bond. Here, we start from known elastic properties of the bond and derive the properties of the repulsive potential between the oxygen and hydrogen atom. The vibrational frequency of the $\mathrm{OH}$-bond is known from infrared (IR) spectroscopy, $f \simeq 1 \times 10^{14} \mathrm{~Hz}$ (derived from the wave number $\tilde{\nu}_{1}=3657 \mathrm{~cm}^{-1}$ of the symmetric stretch [11]). The ratio of the spring constant $k$ and the mass of a hydrogen atom $m=1.67 \times 10^{-27} \mathrm{~kg}$ determines the angular frequency so that the spring constant follows $k=m \omega^{2} \simeq 700 \mathrm{~N} \mathrm{~m}^{-1}$. Since $k \mathcal{L}=7 \times 10^{-8} \mathrm{~N}$, we find the spring constant in atomistic units

$$
k \simeq 1000 \mathrm{kcal} \mathrm{mol}^{-1}(\AA)^{-2} .
$$

The vibrational properties implies a harmonic approximation of the intramolecular potential of the $\mathrm{OH}-$ bond

$$
U_{\text {intra }}(r)=U_{\min }+\frac{1}{2} 1000 \mathrm{kcal} \mathrm{mol}^{-1}\left(r-l_{0}\right)^{2} .
$$

The potential $U_{\text {intra }}$ is the sum of electrostatic potential energy EPE and the repulsion $U_{\mathrm{r}}$ between $\mathrm{O}$ - and $\mathrm{H}$-atoms:

${ }^{1}$ Partial, or atomic, charges are determined both experimentally and computationally. Different theoretical models yield values that differ significantly. We refer the reader to [9] for a detailed discussion of the various computational methods. 


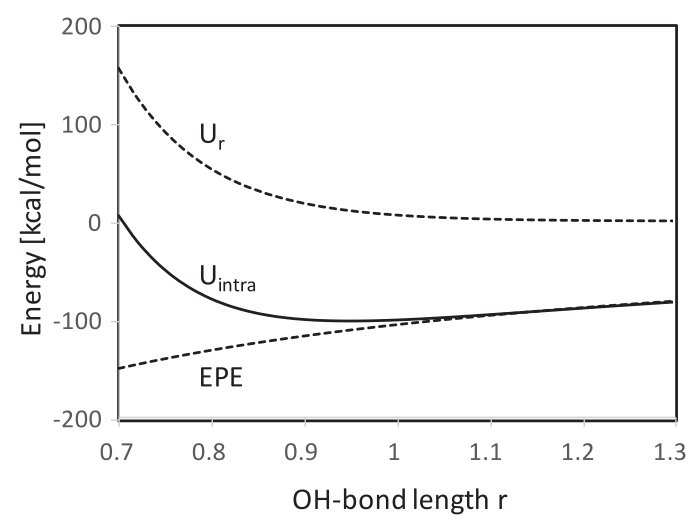

Figure 2. The electrostatic potential energy (EPE), repulsive potential $\left(U_{\mathrm{r}}\right)$, and intramolecular energy $\left(U_{\text {intra }}\right)$ as a function of the $\mathrm{OH}-$ bond length.

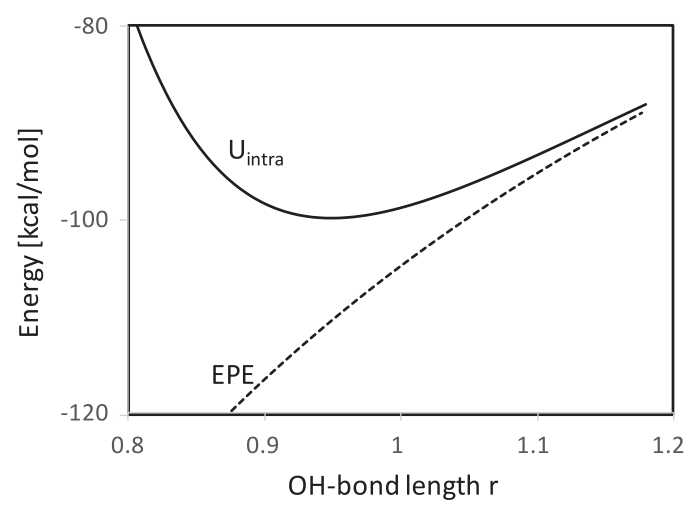

Figure 3. The electrostatic potential energy (EPE), and intramolecular potential $\left(U_{\text {intra }}\right)$ as a function of the OH-bond length.

$$
U_{\text {intra }}(r)=\operatorname{EPE}(r)+U_{\mathrm{r}}(r) .
$$

We assume exponential behavior

$$
U_{\mathrm{r}}(r)=U_{0} \mathrm{e}^{-\kappa r}
$$

We determine the constant $U_{0}$ and $\kappa$ from first and second derivatives at the equilibrium distance $l_{0} \mathrm{~d} U_{\text {intra }} /\left.\mathrm{d} r\right|_{l_{0}}=0$ and $\mathrm{d}^{2} U_{\text {intra }} /\left.\mathrm{d} r^{2}\right|_{l_{0}}=1000 \mathrm{kcal} \mathrm{mol}^{-1}$, and obtain

$$
\kappa=10.7
$$

and

$$
U_{0}=2.8 \times 10^{5} \mathrm{kcal} \mathrm{mol}^{-1} .
$$

Thus, the strength of the repulsion is much larger than that of the Coulomb force $U_{0} \gg \mathcal{U}$, but acts only over a very small range, $\delta r \simeq 1 / \kappa=0.1$. The exponential behavior of the repulsion between $\mathrm{O}$ - and $\mathrm{H}$-atoms is only an approximation, and we expect that the constants depend on the distance $r, U_{0}=U_{0}(r)$ and $\kappa=\kappa(r)$. 


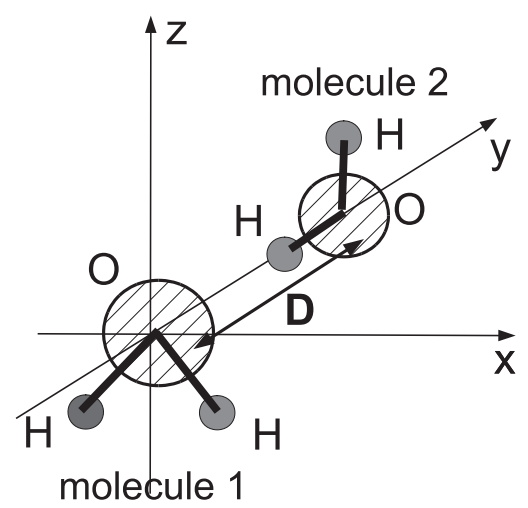

Figure 4. A simplified model of a water dimer; the angle between the $\mathrm{OH}-$ bonds is $90^{\circ}$ and the $\mathrm{OH}$-bond length is 1 . The molecules 1 and 2 are in the $(x, z)$ - and $(y, z)$-planes, respectively. The OO-distance is $D$.

The electrostatic potential energy EPE, the repulsive potential $U_{\mathrm{r}}$, and the total energy $U_{\text {intra }}$ of the OH-bond are shown in figure 2; the electrostatic potential energy EPE and the total energy $U_{\text {intra }}$ of the $\mathrm{OH}$-bond are shown in greater detail in figure 3 . We direct the reader's attention to the narrow range of the $\mathrm{OH}$-bond length, which is necessary since the repulsive potential becomes much larger at smaller distances, e.g., $U_{\mathrm{r}}(0.5) \simeq$ $1300 \mathrm{kcal} \mathrm{mol}^{-1}$. In figure 4, we plot the electrostatic potential energy EPE and the intramolecular potential $U_{\text {intra }}$ over a smaller range of bond length. We find the minimum energy of the $\mathrm{OH}$-bond $U_{\min }=-100 \mathrm{kcal} \mathrm{mol}^{-1}$.

We assume that the water molecule is placed in a region with electric field $E_{\max }$. Because the oxygen and hydrogen are pulled in opposite directions, we use $\delta q=1.2$ and find from equation (5): $F=2.5 \times 10^{-6}=1000 \cdot \delta r$ and find that the $\mathrm{OH}-$ bond is stretched negligibly, $\delta r=2.5 \times 10^{-9}$. Thus, an external force excites the $\mathrm{OH}$-bond only if it is applied periodically with a frequency that corresponds to the natural frequency $\omega$ of the bond, which is the case when water is placed in a microwave oven. The strong frequency dependence is evident in transmission of light: a jar of water is nearly transparent when viewed with visible light (frequency range $4 \times 10^{14} \mathrm{~Hz}<f_{\text {vis }}<8 \times 10^{14} \mathrm{~Hz}$ ) but is opaque when viewed with microwaves (frequency $f_{\text {micro }} \simeq 1 \times 10^{11} \mathrm{~Hz}$ ).

Water dissociates (i.e., the $\mathrm{OH}$-bond breaks) when the temperature is a few thousand kelvin; we use $^{2} T_{\text {dissoc }} \simeq 3000 \mathrm{~K}$ [10]. The corresponding thermal energy follows $\Delta U_{\text {dissoc }}=k_{\mathrm{B}} T_{\text {dissoc }}=6 \mathrm{kcal} \mathrm{mol}^{-1}$. We set the dissociation energy equal to the elastic energy of the OH-bond: $6=1000(\Delta r)^{2} / 2$, and find $\Delta r=0.08$. The value is consistent with the Lindemann criterion $^{3} \Delta r / l_{0} \simeq 0.1$. We note that the variations of the bond lengths are much smaller than length scale associated with repulsive potential, $\delta r, \Delta r<1 / \kappa$, as it should be for consistency of the assumed exponential behavior of the repulsive potential.

\footnotetext{
2 More precisely, the fraction of dissociated water molecules increases as temperature increases. At $3000 \mathrm{~K}$, the fraction is $11 \%$, see, [10].

3 This estimate is consistent with the Lindemann criterion for melting who proposed that melting occurs when the amplitude of the vibrations exceeds $10 \%$ of the atomic spacing. See, e.g., [7], p 268.
} 


\section{Intermolecular forces}

We turn to intermolecular interaction and discuss the hydrogen bond of a water dimer. We simplify the water molecules and assume that the two $\mathrm{OH}$-bonds make a right angle and assume that the equilibrium bond length is $l_{0}=1$. The geometry is shown in figure 4 . We use coordinate systems $(x, y, z)$ such that molecule 1 is in the $(x, z)$-plane. The oxygen atom is at the origin $\vec{r}_{\mathrm{O}}=(0,0,0)$ and the hydrogen atoms: $\vec{r}_{\mathrm{H} \pm}=( \pm 1 / \sqrt{2}, 0,-1 / \sqrt{2})$. Molecule 2 is in the $(y, z)$-plane. We use $D>1$ for the distance between the oxygen atoms. The oxygen atom of molecule 2 is at $\vec{r}_{\mathrm{O}}^{\prime}=(0, D, 0)$, the bonded hydrogen at $\vec{r}_{\mathrm{Hb}}^{\prime}=(0, D-1,0)$, and the unbonded hydrogen at ${\overrightarrow{r^{\prime}}}_{\mathrm{Hu}}=(0, D, 1)$. The empirical value of the OO distance is $L_{0}=2.95$.

We calculate the force on a unit charge $\delta q=1$ at $\vec{r}=(0, y, z)$ due to the charges on molecule 1 . We calculate the $y$-component of force $f_{y}$ : the contribution of the oxygen atom

$$
f_{\mathrm{O}, y}=-264 \mathrm{kcal} \mathrm{mol}^{-1} \AA^{-1} \frac{y}{\left[y^{2}+z^{2}\right]^{3 / 2}}
$$

and the contributions of the two hydrogen atoms:

$$
f_{\mathrm{H}, y}=264 \mathrm{kcal} \mathrm{mol}^{-1} \AA^{-1} \frac{y}{\left[1 / 2+y^{2}+(z+1 / \sqrt{2})^{2}\right]^{3 / 2}} .
$$

The total force follows $f_{y}=f_{\mathrm{O}, y}+f_{\mathrm{H}, y}$, or

$$
\begin{aligned}
f_{y}(y, z)= & 264 \mathrm{kcal} \mathrm{mol}^{-1} \AA^{-1} \\
& \times\left\{\frac{y}{\left[1 / 2+y^{2}+(z+1 / \sqrt{2})^{2}\right]^{3 / 2}}-\frac{y}{\left[y^{2}+z^{2}\right]^{3 / 2}}\right\} .
\end{aligned}
$$

We now calculate the force on molecule 2 due to molecule 1 . The contribution of the oxygen atom:

$$
F_{\mathrm{O}, y}(D)=-211 \mathrm{kcal} \mathrm{mol}^{-1} \AA^{-1}\left\{\frac{D}{\left[D^{2}+1\right]^{3 / 2}}-\frac{1}{D^{2}}\right\},
$$

the contribution of the bonded hydrogen atom:

$$
F_{\mathrm{Hb}, y}(D)=105.5 \mathrm{kcal} \mathrm{mol}^{-1} \AA^{-1}\left\{\frac{D-1}{\left[(D-1)^{2}+1\right]^{3 / 2}}-\frac{1}{(D-1)^{2}}\right\}
$$

and the contribution of the unbonded hydrogen atom:

$$
F_{\mathrm{Hu}, y}(D)=105.5 \mathrm{kcal} \mathrm{mol}^{-1} \AA^{-1}\left\{\frac{D}{\left[1 / 2+D^{2}+(1+1 / \sqrt{2})^{2}\right]^{3 / 2}}-\frac{D}{\left[D^{2}+1\right]^{3 / 2}}\right\} .
$$

The electrostatic force between the water molecules along the $y$-axis follows

$$
F_{y}(D)=F_{\mathrm{O}, y}(D)+F_{\mathrm{Hb}, y}(D)+F_{\mathrm{Hu}, y}(D) .
$$

The forces $F_{\mathrm{O}, y}, F_{\mathrm{Hb}, y}, F_{\mathrm{Hu}, y}$, and $F_{y}$ as a function of the OO-distance are shown in figure 5 . We conclude that the forces exerted on the oxygen atom and the unbonded hydrogen atom approximately cancel out each other; and the force between the two water molecules is largely due to the bonded hydrogen atom.

We plot $\log \left(\left|F_{y}\right|\right)$ versus $\log (D)$ in figure 6; the slope of the dashed line is 5 and the intercept is 7.8. We find the power-law behavior of the attractive force induced by the 


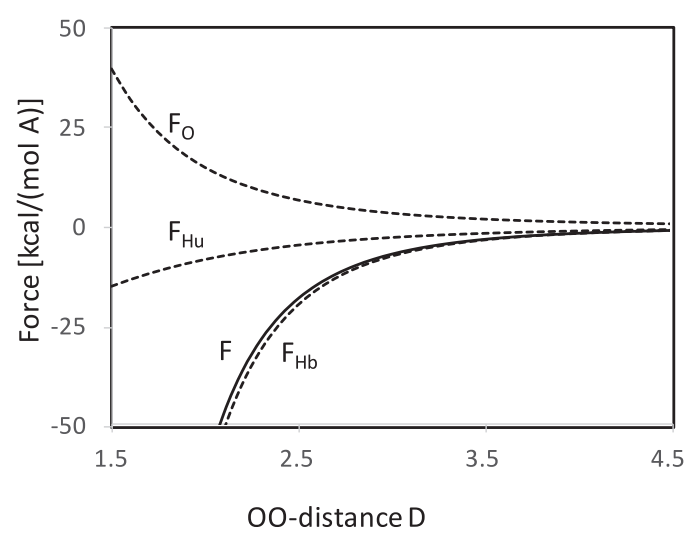

Figure 5. The electrostatic force along the $y$-axis on the oxygen atom $(\mathrm{O})$, bonded hydrogen atom $(\mathrm{Hb})$, unbonded hydrogen atom ( $\mathrm{Hu}$ ) (all dashed), and the net electrostatic force along (solid) as a function of the OO-distance $D$.

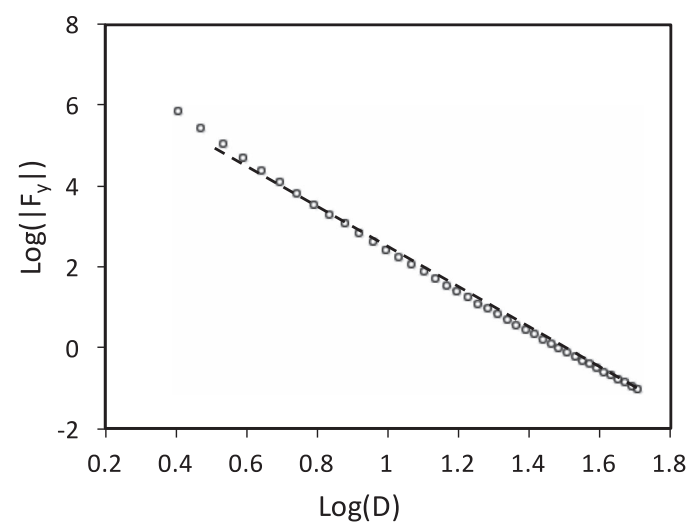

Figure 6. The net force along the $y$-axis as function of the OO-distance in a doublelogarithmic plot; the dashed line has slope 5 and intercept 7.8.

hydrogen bond for $D>D_{c}=2$ :

$$
F_{y}(D) \simeq-52 \mathrm{kcal} \mathrm{mol}^{-1} \AA^{-1}\left(\frac{D_{c}}{D}\right)^{5}, \quad D>D_{c} .
$$

We conclude that the attractive electrostatic interaction between the two water molecules in a water molecule decays faster than the inverse-square dependence Coulomb's law, which reflects the electrically neutral character of water molecules. We emphasize that the exponent $n=5$ is not universal and applies only to the particular geometry of the dimer. The prefactors in equations (8) and (23)are of the same order $\left(330 \mathrm{kcal}(\mathrm{mol} \AA)^{-1} \operatorname{versus} 52 \mathrm{kcal}(\mathrm{mol} \AA)^{-1}\right)$ so that the strengths of the forces are similar. We obtain the attractive Coulomb force between the water molecules near the equilibrium distance $L_{0}, F_{\mathrm{C}, y}\left(L_{0}\right) \simeq-6.8 \mathrm{kcal}(\mathrm{mol} \AA)^{-1}$.

We find the electrostatic potential energy of a unit charge $\delta q=1$ at $\vec{r}=(0, y, z)$ in the electric field produced by the water molecule 1 . The contribution of the oxygen atom: 


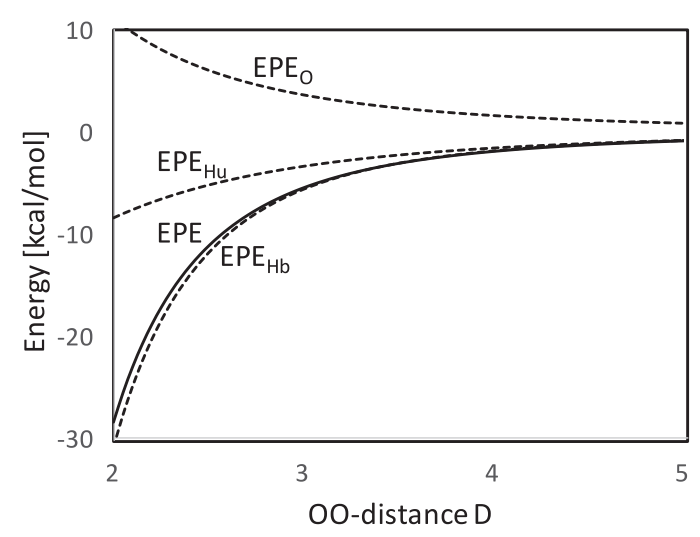

Figure 7. The electrostatic potential of the oxygen atom $(\mathrm{O})$, bonded hydrogen atom $(\mathrm{Hb})$, unbonded hydrogen atom $(\mathrm{Hu})$ (all dashed), and the total electrostatic potential energy as a function of the OO-distance $D$.

$$
u_{\mathrm{O}}=-264 \mathrm{kcal} \mathrm{mol}^{-1} \frac{1}{\sqrt{y^{2}+z^{2}}}
$$

and the two hydrogen atoms:

$$
u_{\mathrm{H}}=264 \mathrm{kcal} \mathrm{mol}^{-1} \frac{1}{\sqrt{1 / 2+y^{2}+(z+1 / \sqrt{2})^{2}}} .
$$

The total electrostatic potential energy $u_{\mathrm{O}}+u_{\mathrm{H}}$ follows

$$
u=264 \mathrm{kcal} \mathrm{mol}^{-1}\left\{\frac{1}{\sqrt{1 / 2+y^{2}+(z+1 / \sqrt{2})^{2}}}-\frac{1}{\sqrt{y^{2}+z^{2}}}\right\} .
$$

We now calculate the electrostatic potential energy of the water molecule 2 in the electric field produced by molecule 1 . The contribution of the oxygen atom:

$$
\mathrm{EPE}_{\mathrm{O}}=-211 \mathrm{kcal} \mathrm{mol}^{-1}\left\{\frac{1}{\left[1+D^{2}\right]^{1 / 2}}-\frac{1}{D}\right\}
$$

the bonded hydrogen:

$$
\mathrm{EPE}_{\mathrm{Hb}}=105.5 \mathrm{kcal} \mathrm{mol}^{-1}\left\{\frac{1}{\left[1+(D-1)^{2}\right]^{1 / 2}}-\frac{1}{D-1}\right\}
$$

and the unbonded hydrogen:

$\mathrm{EPE}_{\mathrm{Hu}}=105.5 \mathrm{kcal} \mathrm{mol}^{-1}\left\{\frac{1}{\left[1 / 2+D^{2}+(1+1 / \sqrt{2})^{2}\right]^{1 / 2}}-\frac{1}{\left[D^{2}+1\right]^{1 / 2}}\right\}$.

The total potential energy of the electrostatic interaction between the two water molecules follows

$$
\mathrm{EPE}(D)=\operatorname{EPE}_{\mathrm{O}}(D)+\operatorname{EPE}_{\mathrm{Hb}}(D)+\operatorname{EPE}_{\mathrm{Hu}}(D) .
$$

We plot $\mathrm{EPE}_{\mathrm{O}}, \mathrm{EPE}_{\mathrm{Hb}}, \mathrm{EPE}_{\mathrm{Hu}}$, and $\mathrm{EPE}$ as a function of the OO-distance $D$ in figure 7 . We find that the total electrostatic potential energy is dominated by the potential energy of the bonded hydrogen atom, $\mathrm{EPE} \simeq \mathrm{EPE}_{\mathrm{Hb}}$, which is, of course, consistent with the analogous 


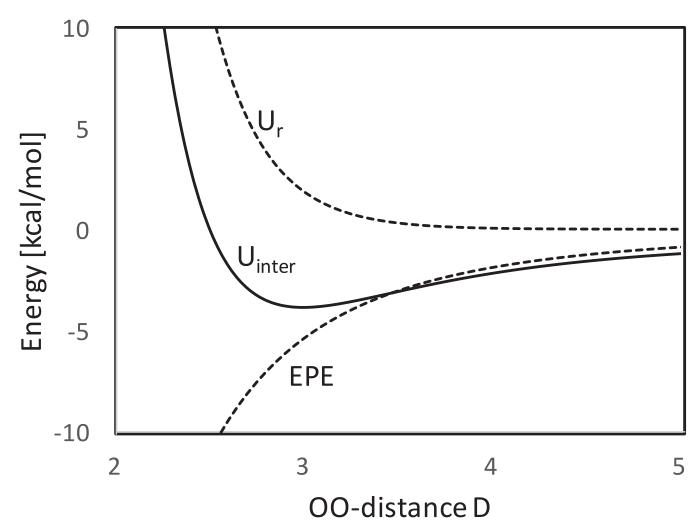

Figure 8. The total electrostatic potential energy EPE, the repulsive potential $U_{\mathrm{r}}$, and the intermolecular energy $U_{\text {inter }}$ as a function of the OO-distance.

property of the electrostatic force between water molecules. We find the electrostatic potential energy at the equilibrium distance $L_{0}: \operatorname{EPE}\left(L_{0}\right)=-5.7 \mathrm{kcal} \mathrm{mol}^{-1}$.

We assume that the repulsion between the oxygen and hydrogen atoms of the hydrogen bond has exponential behavior, see equation (13)

$$
U_{\mathrm{r}}^{\prime}(D)=U_{0}^{\prime} \mathrm{e}^{-\kappa^{\prime} D} .
$$

We expect that the repulsive potential is weaker at larger distances

$$
U_{0}^{\prime}=U_{0} \frac{l_{0}}{L_{0}}=9.1 \times 10^{4}
$$

and dependence on the distance is softer

$$
\kappa^{\prime}=\kappa \frac{l_{0}}{L_{0}}=3.6
$$

The repulsive potential energy at the equilibrium distance follows $U_{\mathrm{ex}}\left(L_{0}\right)=+2.2 \mathrm{kcal} \mathrm{mol}^{-1}$.

The intermolecular potential energy of the hydrogen bond in the water dimer follows

$$
U_{\text {inter }}(D)=\operatorname{EPE}(D)+U_{\mathrm{r}}^{\prime}(D)
$$

We plot the electrostatic potential energy EPE, the repulsive potential between water molecules $U_{\mathrm{r}}^{\prime}$, and the intermolecular energy $U_{\text {inter }}$ as a function of the OO-distance in figure 8 . We require that the equilibrium distance $L_{0}$ is the local minimum of the intermolecular potential. We find the repulsive force: $F_{\mathrm{r}}\left(L_{0}\right)=-\mathrm{d} U_{\mathrm{r}} /\left.\mathrm{d} D\right|_{L_{0}}=+6.6 \mathrm{kcal}(\mathrm{mol} \AA)^{-1}$. The net force follow $F_{\text {total }}\left(L_{0}\right)=F_{\mathrm{C}, y}\left(L_{0}\right)+F_{\mathrm{r}}\left(L_{0}\right) \simeq-6.8 \mathrm{kcal}(\mathrm{mol} \AA)^{-1}+$ $6.6 \mathrm{kcal}(\mathrm{mol} \AA)^{-1} \simeq 0$, as it should.

We find the repulsive potential at the equilibrium distance $U_{\mathrm{r}}\left(L_{0}\right)=2.2 \mathrm{kcal} \mathrm{mol}^{-1}$ so that the inrermolecular potential has the minimum $U_{\text {inter }}\left(L_{0}\right)=-5.7 \mathrm{kcal} \mathrm{mol}^{-1}+$ $2.2 \mathrm{kcal} \mathrm{mol}^{-1}$ so that

$$
U_{\min }^{\prime}=-3.5 \mathrm{kcal} \mathrm{mol}^{-1} \text {. }
$$

This energy corresponds to the binding energy (or 'bond strength') of the hydrogen bond of the water dimer; a quantum-mechanical calculation yield $\Delta E_{\mathrm{H}-\text { bond }}=3.4 \mathrm{kcal} \mathrm{mol}^{-1}$ [12]. Thus, our approximate theory gives a surprisingly accurate result, and validates our use of a phenomenological form for repulsion between charges. 
We use a harmonic approximation of the intermolecular interaction

$$
U_{\text {inter }}(D)=U_{\min }^{\prime}+\frac{1}{2} k^{\prime}\left(D-L_{0}\right)^{2} .
$$

The spring constant follows from the second derivative $k^{\prime}=\mathrm{d}^{2} U_{\text {inter }} /\left.\mathrm{d} D^{2}\right|_{L_{0}}$

$$
k^{\prime} \simeq 12 \mathrm{kcal} \mathrm{mol}^{-1}(\AA)^{-2} .
$$

We conclude that the intra-molecular $\mathrm{OH}$-bond is much stiffer than the inter-molecular hydrogen bond.

We use the Lindemann criterion to find an estimate for the temperature when the water dimer breaks up. We find $\Delta D \simeq 0.1 L_{0}=0.3$ and find $\Delta U_{\text {inter }} \simeq(1 / 2)$ $12 \mathrm{kcal} \mathrm{mol}^{-1}(0.3)^{2}=0.5 \mathrm{kcal} \mathrm{mol}^{-1}$. The corresponding temperature follows $T_{\text {dissoc }}^{\prime}=\Delta U_{\text {inter }} / k_{\mathrm{B}}=\left(0.5 \mathrm{kcal} \mathrm{mol}^{-1}\right) /\left[0.002 \mathrm{kcal}(\mathrm{mol} \mathrm{K})^{-1}\right]=250 \mathrm{~K}$, or approximately the melting temperature of ice $\left(T_{\text {melt }}=273 \mathrm{~K}\right)$. We point out that the rather accurate predicted value of the melting temperature of water is due to fortuitous choice of parameter, in particular the choice of partial charges of hydrogen and oxygen atoms. The result $T_{\text {dissoc }}^{\prime} \simeq T_{\text {melt }}$ is consistent with the view that melting is associated with breaking of bonds between water molecules that give rise to formation of ice crystals.

\section{Discussion}

A recent paper in The Physics Teacher pointed out that macroscopic application of electrostatics generally necessitates that the system's parameters (mass, charge, size, etc) are finetuned. In fact, Coulomb forces are generally not noticeable at length scales of $1 \mathrm{~cm}$ or longer because macroscopic objects typically have zero net charge. In contrast, positive and negative charges are separated in molecules, which explains why Coulomb forces are dominant at the molecular level. While electrons must be described by the laws of quantum mechanics at lengths of molecules (nano-meter), we propose a phenomenological treatment in terms of partial charges and a repulsive potential.

The connection with the macroscopic world is then achieved by multiplying molecular interaction energies with Avogadro's number and calculate molar heat In this way, molecular systems are excellent examples to illustrate principles of electrostatics, since no 'fine-tuning' of parameters is necessary.

We discuss the properties of the $\mathrm{OH}$-bond of a water molecule and the hydrogen bond of a water dimer, and showed that the description of the charge repulsion allows to derive properties of the molecular systems that are surprisingly close to their empirical values. We relate the properties of the intra- and inter-molecular forces to thermal properties of water.

A phenomenological treatment for the quantum-mechanical repulsion is key for our calculation, and we use empirical values of the $\mathrm{OH}$ - and hydrogen-bond lengths to find parameters that characterize this 'effective' potential. We are familiar with a similar situation from Newtonian mechanics, where the summation of small elastic compression forces between two objects (for example, two blocks) is treated as 'normal force.' There is no general expression for this force and its value is determined as part of the solution of the mechanical problem (the net force along a direction perpendicular to the contact surface must be zero).

An emphasis on 'chemical' problems is suitable in particular for the algebra-based sequence. Most students also take general and organic chemistry, as well as biochemistry, and are thus familiar with molecular models. While some of the calculations presented here are beyond the level of such a course, it is straightforward to simplify the mathematics (e.g., by 
using graphical methods rather than calculus) to make it suitable for classroom use. The use of atomistic units (e.g., $\mathrm{kcal} \mathrm{mol}^{-1}$ for energy) is particularly helpful to make connections with macroscopic properties.

\section{Acknowledgments}

I would like to thank John Masnovi (Chemistry Department, Cleveland State University) for a careful reading of the manuscript and helpful suggestions.

\section{References}

[1] http://nextgenscience.org

[2] Feynman R P, Leighton R B and Sands M 1964 The Feynman Lectures vol 2 (Reading MA: Addison-Wesley)

[3] Still commonly referred to as 'Sears Tower'. See: http://theskydeck.com/for-kids/fun-facts; accessed 22/09/2016

[4] Morse R A 2016 Phys. Teach. 54 200-2

[5] Chaikin P M and Lubensky T C 1995 Principles of Condensed Matter Physics (New York: Cambridge University Press) $\mathrm{p} 1$

[6] See e.g., Berry R S, Rice S A and Ross A 2002 Matter in Equilibrium-Part I (New York: Oxford University Press) ch 6

[7] Tabor D 1991 Gases, Liquids, and Solids, and Other States of Matter 3rd edn (New York: Cambridge University Press)

[8] Ashcroft N W and Mermin N D 1976 Solid State Physics (Philadelphia, PA: Holt-Saunders) p 2

[9] Martin F and Zipse F 2004 J. Comput. Chem. 26 97-105

[10] Langmuir A $1906 \mathrm{~J}$. Am. Chem. Soc. 28 1357-79

[11] Silbey R J, Alberty R A and Bawendi M G 2005 Physical Chemistry 4th edn (New York: Wiley) p 487

[12] Keutsch F N and Saykally R J 2001 Proc. Natl Acad. Sci. USA 98 10533-40 